\title{
Mechanism of IncRNA H19 in the Regulation of Lipid Accumulation and Inflammatory Response in Macrophages
}

\author{
Xue-Mei Wang ( $\nabla$ wxuemei1983@sina.com ) \\ Xiao-Ming Gao \\ First Affliated Hospital of Xinjiang Medical University \\ Fen Liu \\ First Affiliated Hospital of Xinjiang Medical University \\ Ying Cao \\ XI' AN Medical University \\ Jie-Ying Wang \\ XI'AN Medical University \\ Yuan Xing \\ XI'AN Medical University
}

\section{Research}

Keywords: atherosclerosis, IncRNA H19, NF-kB, lipids accumulation, cholesterol reverse transport

Posted Date: August 21st, 2020

DOl: https://doi.org/10.21203/rs.3.rs-27706/v2

License: (9) This work is licensed under a Creative Commons Attribution 4.0 International License. Read Full License 
The authors have withdrawn this preprint from Research Square 